\title{
Research Article \\ Comparison Theorems for the Multidimensional BDSDEs and Applications
}

\author{
Bo Zhu' ${ }^{1}$ and Baoyan Han ${ }^{2}$ \\ ${ }^{1}$ School of Mathematic and Quantitative Economics, Shandong University of Finance and Economics, \\ Jinan 250014, China \\ 2 Shandong University of Art and Design, Jinan 250014, China
}

Correspondence should be addressed to Bo Zhu, zhubo207@163.com

Received 16 December 2011; Accepted 16 February 2012

Academic Editor: A. A. Soliman

Copyright (C) 2012 B. Zhu and B. Han. This is an open access article distributed under the Creative Commons Attribution License, which permits unrestricted use, distribution, and reproduction in any medium, provided the original work is properly cited.

A class of backward doubly stochastic differential equations (BDSDEs) are studied. We obtain a comparison theorem of these multidimensional BDSDEs. As its applications, we derive the existence of solutions for this multidimensional BDSDEs with continuous coefficients. We can also prove that this solution is the minimal solution of the BDSDE.

\section{Introduction}

Backward stochastic differential equations (BSDEs in short) were first introduced in [1] in order to give a probabilistic interpretation (Feynman-Kac formula) for the solutions of semilinear parabolic PDEs, one can see $[2,3]$. Moreover, BSDEs have been considered with great interests because for their connections with mathematical finance [4] as well as stochastic optimal control and stochastic games $[5,6]$. As we know, the comparison theorem is a very useful result in the theory of BSDEs, for example, it can be used to study viscosity solutions of partial differential equations. In 1992, Peng [2] gave the comparison of the onedimensional BSDE. In 1994, Christel Geiß and Ralf Manthey [7] proved the comparison theorems for stochastic differential equations in finite and infinite dimensions.

After they introduced the theory of BSDEs, Pardoux and Peng [8] in 1994 brought forward a new kind of BSDEs, that is a class of backward doubly stochastic differential equations (BDSDEs in short) with two different directions of stochastic integrals, that is, the equations involve both a standard (forward) stochastic integral $d W_{t}$ and a backward stochastic integral $d B_{t}$. They have proved the existence and uniqueness of solutions to BDSDEs under uniformly Lipschitz conditions on coefficients. That is, for a given terminal time $T>0$, under the uniformly Lipschitz assumptions on coefficients $f, g$, for any square 
integrable terminal value $\xi$, the following BDSDE has a unique solution pair $\left(y_{t}, z_{t}\right)$ in the interval $[0, T]$ :

$$
y_{t}=\xi+\int_{t}^{T} f\left(s, y_{s}, z_{s}\right) d s+\int_{t}^{T} g\left(s, y_{s}, z_{s}\right) d B_{s}-\int_{t}^{T} z_{s} d W_{s}
$$

Pardoux and Peng [8] showed that BDSDEs can produce a probabilistic representation for certain quasilinear stochastic partial differential equations (SPDEs). Recently, [9] Zhang and Zhao, [10] Zhu and Han studied the infinite horizon BDSDEs. Many researchers do their work in this area (cf. [11-18] and the references therein). As we know, the comparison theorem is a very useful result in the theory of BDSDEs, for example, it can be used to study viscosity solutions of SPDEs [19]. Shi et al. proved the comparison theorem of these BDSDEs, but they studied the one-dimensional BDSDEs.

In this paper, we will prove the comparison theorem of the multidimensional BDSDEs. Then we study the multidimensional BDSDEs with continuous coefficients as an application of the comparison theorem.

The paper is organized as follows: in Section 2 we introduce some preliminaries and notations; in Section 3 we prove the comparison theorem of multidimensional BDSDEs; at the end, we give the multi-dimension BDSDEs with continuous coefficients in Section 4.

\section{Preliminaries: The Existence and Uniqueness of BDSDEs and an Extension of Itô Formula}

Notation 1. The Euclidean norm of a vector $x \in R^{k}$ will be denoted by $|x|$, and for a $d \times k$ matrix $A$, we define $\|A\|=\sqrt{\operatorname{Tr} A A^{*}}$, where $A^{*}$ is the transpose of $A$. We also define $(\alpha, \beta)$ as the inner product of $\alpha$ and $\beta$.

For $a \in R^{k}$, let $a_{i}$ be the $i$ th component of $a ; c \in R^{k \times d}$, let $c_{i}$ be the $i$ th row of $c$, let $c_{i, j}$ be an element for the $i$ th row and the $j$ th column of $c$. For $a^{1}, a^{2} \in R^{k}$, we define

$$
a^{1} \geq a^{2} \Longleftrightarrow a_{j}^{1} \geq a_{j}^{2}, \quad j=1,2, \ldots, k
$$

Let $(\Omega, \mathcal{F}, P)$ be a probability space and let $T$ be an arbitrarily fixed positive constant throughout this paper. Let $\left\{W_{t} ; 0 \leq t \leq T\right\}$ and $\left\{B_{t} ; 0 \leq t \leq T\right\}$ be two mutually independent standard Brownian motions with values in $R^{d}$ and $R^{l}$, respectively, defined on $(\Omega, \mathcal{F}, P)$. Let $\mathcal{N}$ denote the class of $P$-null sets of $\mathcal{F}$. For each $t \in[0, T]$, we define

$$
\mathcal{F}_{t} \doteq \mathcal{F}_{t}^{W} \vee \mathscr{F}_{t, T^{\prime}}^{B}
$$

where for any process $\left\{\eta_{t}\right\}, \boldsymbol{F}_{s, t}^{\eta}=\sigma\left\{\eta_{r}-\eta_{s} ; s \leq r \leq t\right\} \vee \mathcal{N}, \mathbb{F}_{t}^{\eta}=\mathbb{F}_{0, t}^{\eta}$.

Note that the collection $\left\{\mathscr{f}_{t} ; t \in[0, T]\right\}$ is neither increasing nor decreasing, and it does not constitute a filtration.

For any $n \in N$, let $M^{2}\left(0, T ; R^{n}\right)$ denote the set of (classes of $d P \otimes d t$ a.e. equal) $n$ dimensional jointly measurable stochastic processes $\left\{\varphi_{t} ; t \in[0, T]\right\}$ which satisfy

(i) $\|\varphi\|_{M^{2}}^{2}:=E \int_{0}^{T}\left|\varphi_{t}\right|^{2} d t<\infty$;

(ii) $\varphi_{t}$ is $\mathcal{F}_{t}$-measurable, for a.e. $t \in[0, T]$. 
Similarly, we denote by $S^{2}\left([0, T] ; R^{n}\right)$ the set of $n$-dimensional continuous stochastic processes $\left\{\varphi_{t} ; t \in[0, T]\right\}$ which satisfy

(iii) $\|\varphi\|_{S^{2}}^{2}:=E\left(\sup _{0 \leq t \leq T}\left|\varphi_{t}\right|^{2}\right)<\infty$;

(iv) $\varphi_{t}$ is $\mathcal{F}_{t}$-measurable, for any $t \in[0, T]$.

Obviously, $M^{2}\left(0, T ; R^{n}\right)$ and $S^{2}\left([0, T] ; R^{n}\right)$ are Hilbert spaces.

Let

$$
f: \Omega \times[0, T] \times R^{k} \times R^{k \times d} \longrightarrow R^{k}, \quad g: \Omega \times[0, T] \times R^{k} \times R^{k \times d} \longrightarrow R^{k \times l}
$$

be jointly measurable and such that for any $(y, z) \in R^{k} \times R^{k \times d}$.

(H1) $f(\cdot, y, z) \in M^{2}\left(0, T ; R^{k}\right), g(\cdot, y, z) \in M^{2}\left(0, T ; R^{k \times l}\right)$.

(H2) There exist constants $C>0$ and $0<\alpha<1$ such that for any $(\omega, t) \in \Omega \times[0, T]$, $\left(y_{1}, z_{1}\right),\left(y_{2}, z_{2}\right) \in R^{k} \times R^{k \times d}$,

$$
\begin{aligned}
\left|f\left(t, y_{1}, z_{1}\right)-f\left(t, y_{2}, z_{2}\right)\right|^{2} & \leq C\left(\left|y_{1}-y_{2}\right|^{2}+\left\|z_{1}-z_{2}\right\|^{2}\right) \\
\left\|g\left(t, y_{1}, z_{1}\right)-g\left(t, y_{2}, z_{2}\right)\right\|^{2} & \leq C\left|y_{1}-y_{2}\right|^{2}+\alpha\left\|z_{1}-z_{2}\right\|^{2} .
\end{aligned}
$$

Given $\xi \in L^{2}\left(\Omega, \mp_{T}, P ; R^{k}\right)$, we consider the following backward doubly stochastic differential equation:

$$
Y_{t}=\xi+\int_{t}^{T} f\left(s, Y_{s}, Z_{s}\right) d s+\int_{t}^{T} g\left(s, Y_{s}, Z_{s}\right) d B_{s}-\int_{t}^{T} Z_{s} d W_{s}, \quad 0 \leq t \leq T
$$

Note that the integral with respect to $\left\{B_{t}\right\}$ is a "backward Itô integral" and the integral with respect to $\left\{W_{t}\right\}$ is a standard forward Itô integral. The forward integrals were defined in [20] Da Prato and Zabczyk and [21] D. Nualart, and E. Pardoux. To see the backward one, for any $s \geq 0$ let $\{h(s)\}_{s \geq 0}$ be a stochastic process such that $\{h(s)\}$ is $\mathcal{F}_{s}$ measurable, and locally square integrable, that is, for any $0 \leq a \leq b<\infty, \int_{a}^{b}\|h(s)\|^{2} d s<\infty$ almost surely. Since $\mathcal{F}_{s}$ is a backward filtration with respect to $B$, so from the one-dimensional backward Itô's integral and relation with forward integral, for $0 \leq T \leq T^{\prime}$, we have $\int_{t}^{T} h(s) d B s=-\int_{T^{\prime}-T}^{T^{\prime}-t} h\left(T^{\prime}-s\right) d \widehat{B}_{s}$ a.s, where $\widehat{B}_{S}=B_{T^{\prime}-s}-B_{T^{\prime}}$.

Definition 2.1. A pair of processes $(y, z): \Omega \times[0, T] \rightarrow R^{k} \times R^{k \times d}$ is called a solution of BDSDE (2.5), if $(y, z) \in S^{2}\left([0, T] ; R^{k}\right) \times M^{2}\left(0, T ; R^{k \times d}\right)$ and satisfies BDSDE (2.5).

Proposition 2.2. Under conditions (H1) and (H2), BDSDE (2.5) has a unique solution $(Y, Z) \in$ $S^{2}\left([0, T] ; R^{k}\right) \times M^{2}\left(0, T ; R^{k \times d}\right)$.

Proposition 2.3. Let $\alpha \in S^{2}\left([0, T] ; R^{k}\right), \beta \in M^{2}\left(0, T ; R^{k}\right), \gamma \in M^{2}\left(0, T ; R^{k \times l}\right), \delta \in M^{2}\left(0, T ; R^{k \times d}\right)$ satisfy

$$
\alpha_{t}=\alpha_{0}+\int_{0}^{t} \beta_{s} d s+\int_{0}^{t} \gamma_{s} d B_{s}+\int_{0}^{t} \delta_{s} d W_{s}, \quad 0 \leq t \leq T .
$$


Then

$$
\begin{aligned}
\left|\alpha_{t}\right|^{2}= & \left|\alpha_{0}\right|^{2}+2 \int_{0}^{t}\left(\alpha_{s}, \beta_{s}\right) d s+2 \int_{0}^{t}\left(\alpha_{s}, \gamma_{s} d B_{s}\right)+2 \int_{0}^{t}\left(\alpha_{s}, \delta_{s} d W_{s}\right) \\
& -\int_{0}^{t}\left\|\gamma_{s}\right\|^{2} d s+\int_{0}^{t}\left\|\delta_{s}\right\|^{2} d s \\
E\left|\alpha_{t}\right|^{2}= & E\left|\alpha_{0}\right|^{2}+2 E \int_{0}^{t}\left(\alpha_{s}, \beta_{s}\right) d s-E \int_{0}^{t}\left\|\gamma_{s}\right\|^{2} d s+E \int_{0}^{t}\left\|\delta_{s}\right\|^{2} d s .
\end{aligned}
$$

This two propositions were derived in [8].

\section{Comparison Theorem of Multidimensional BDSDEs}

In this section, we will prove the comparison theorem of the multidimensional BDSDEs. Firstly, we give the definition of the indicator function for the positive and negative parts.

Definition 3.1. If $f: \Omega \mapsto \bar{R}$, then the positive part of $f$ is defined by the formula $f^{+} \doteq$ $\max \{f, 0\}$. Similarly, the negative part of $f$ is defined as $f^{-} \doteq \max \{-f, 0\}=-\min \{f, 0\}$

Note that both $f^{+}$and $f^{-}$are nonnegative functions.

We consider the following $k$-dimensional BDSDEs: $(0 \leq t \leq T)$

$$
\begin{aligned}
& y_{t}^{1}=\xi^{1}+\int_{t}^{T} f^{1}\left(s, y_{s}^{1}, z_{s}^{1}\right) d s+\int_{t}^{T} g\left(s, y_{s}^{1}, z_{s}^{1}\right) d B_{s}-\int_{t}^{T} z_{s}^{1} d W_{s}, \\
& y_{t}^{2}=\xi^{2}+\int_{t}^{T} f^{2}\left(s, y_{s}^{2}, z_{s}^{2}\right) d s+\int_{t}^{T} g\left(s, y_{s}^{2}, z_{s}^{2}\right) d B_{s}-\int_{t}^{T} z_{s}^{2} d W_{s}
\end{aligned}
$$

where $\xi^{1}, \xi^{2} \in L^{2}\left(\Omega, \mathcal{F}_{T}, P ; R^{k}\right), f^{1}(\omega, t, y, z), f^{2}(\omega, t, y, z): \Omega \times[0, T] \times R^{k} \times R^{k \times d} \rightarrow R^{k}$, $g(\omega, t, y, z): \Omega \times[0, T] \times R^{k} \times R^{k \times d} \rightarrow R^{k \times l}$. We assume $\xi^{1}, \xi^{2}$ and $f^{1}, f^{2}, g$ satisfy the following conditions:

(i) $\xi^{1} \geq \xi^{2} P$-a.s.;

(ii) For all $j=1,2, \ldots, k$, for all $(\omega, t) \in \Omega \times[0, T], y^{1}, y^{2} \in R^{k}, z^{1}, z^{2} \in R^{k \times d}, y_{j}^{1}=y_{j}^{2}$, $z_{j}^{1}=z_{j}^{2}, y_{l}^{1} \geq y_{l}^{2}, l \neq j$

$$
f_{j}^{1}\left(\omega, t, y^{1}, z^{1}\right) \geq f_{j}^{2}\left(\omega, t, y^{2}, z^{2}\right)
$$

(iii) for all $(\omega, t) \in \Omega \times[0, T], y^{1}, y^{2} \in R^{k}, z^{1}, z^{2} \in R^{k \times d}$

$$
\left|f_{j}^{i}\left(t, y^{1}, z^{1}\right)-f_{j}^{i}\left(t, y^{2}, z^{2}\right)\right|^{2} \leq C\left(\left|y^{1}-y^{2}\right|^{2}+\left\|z^{1}-z^{2}\right\|^{2}\right), \quad i=1,2
$$



such that

(iv) for all $(\omega, t, y, z) \in \Omega \times[0, T] \times R^{k} \times R^{k \times d}, \exists h(\omega, t, \mu, v): \Omega \times[0, T] \times R \times R^{d} \rightarrow R^{l}$

$$
\begin{gathered}
g_{j}(\omega, t, y, z)=h_{j}\left(\omega, t, y_{j}, z_{j}\right), \\
\left|h_{j}\left(t, y_{j}^{1}, z_{j}^{1}\right)-h_{j}\left(t, y_{j}^{2}, z_{j}^{2}\right)\right|^{2} \leq C\left|y_{j}^{1}-y_{j}^{2}\right|^{2}+\alpha\left|z_{j}^{1}-z_{j}^{2}\right|^{2},
\end{gathered}
$$

where $C>0,0<\alpha<1$ are two constants.

From Proposition 2.2, under conditions (iii) and (iv), there exist two pairs of measurable processes $\left(y^{1}, z^{1}\right) \in S^{2}\left([0, T] ; R^{k}\right) \times M^{2}\left(0, T ; R^{k \times d}\right)$ and $\left(y^{2}, z^{2}\right) \in S^{2}\left([0, T] ; R^{k}\right) \times$ $M^{2}\left(0, T ; R^{k \times d}\right)$ satisfying BDSDEs (3.1), respectively.

Theorem 3.2. Assume the conditions (i)-(iv) hold, let $\left(y^{1}, z^{1}\right)$ and $\left(y^{2}, z^{2}\right)$ be solutions of BDSDEs (3.1), respectively. Then $y_{t}^{1} \geq y_{t}^{2}$, a.s. for all $t \in[0, T]$.

Proof. for all $\varepsilon>0$, we define a function $\phi_{\varepsilon}(y): R \rightarrow R$

$$
\phi_{\varepsilon}(y)= \begin{cases}y^{2}, & y \leq 0 \\ y^{2}-\frac{1}{6 \varepsilon} y^{3}, & 0 \leq y \leq 2 \varepsilon \\ 2 \varepsilon y-\frac{4}{3} \varepsilon^{2}, & y \geq 2 \varepsilon .\end{cases}
$$

Obviously, $\phi_{\varepsilon}(y) \in C^{2}(R)$ and $\phi_{\varepsilon}^{\prime \prime}(y)$ is bounded, for all $y \in R$,

$$
\phi_{\varepsilon}(y) \longrightarrow\left|y^{-}\right|^{2}, \quad \phi_{\varepsilon}^{\prime \prime}(y) \longrightarrow-2 y^{-}, \quad \phi_{\varepsilon}^{\prime \prime}(y) \longrightarrow 2 \mathbf{I}_{\{y \leq 0\}}, \quad \text { when } \varepsilon \rightarrow 0 .
$$

We set

$$
\bar{y}_{t}=y_{t}^{1}-y_{t}^{2}, \quad \bar{z}_{t}=z_{t}^{1}-z_{t}^{2}, \quad \bar{\xi}=\xi^{1}-\xi^{2} .
$$

Then $\left(\bar{y}_{t}, \bar{z}_{t}\right)$ satisfy the following BDSDE:

$$
\bar{y}_{t}=\bar{\xi}+\int_{t}^{T}\left[f^{1}\left(s, y_{s}^{1}, z_{s}^{1}\right)-f^{2}\left(s, y_{s}^{2}, z_{s}^{2}\right)\right] d s+\int_{t}^{T}\left[g\left(s, y_{s}^{1}, z_{s}^{1}\right)-g\left(s, y_{s}^{2}, z_{s}^{2}\right)\right] d B_{s}-\int_{t}^{T} \bar{z}_{s} d W_{s} .
$$

Applying Itô's formula to $\phi_{\varepsilon}\left(\bar{y}_{j}(t)\right)$, we get

$$
\begin{aligned}
\phi_{\varepsilon}\left(\bar{y}_{j}(t)\right)= & \phi_{\varepsilon}\left(\bar{\xi}_{j}\right)+\int_{t}^{T} \phi_{\varepsilon}^{\prime}\left(\bar{y}_{j}(s)\right)\left[f_{j}^{1}\left(s, y_{s}^{1}, z_{s}^{1}\right)-f_{j}^{2}\left(s, y_{s}^{2}, z_{s}^{2}\right)\right] d s \\
& +\int_{t}^{T} \phi_{\varepsilon}^{\prime}\left(\bar{y}_{j}(s)\right)\left[g_{j}\left(s, y_{s}^{1}, z_{s}^{1}\right)-g_{j}\left(s, y_{s}^{2}, z_{s}^{2}\right)\right] d B_{s}-\int_{t}^{T} \phi_{\varepsilon}^{\prime}\left(\bar{y}_{j}(s)\right) \bar{z}_{j}(s) d W_{s} \\
& +\frac{1}{2} \int_{t}^{T} \phi_{\varepsilon}^{\prime \prime}\left(\bar{y}_{j}(s)\right)\left|g_{j}\left(s, y_{s}^{1}, z_{s}^{1}\right)-g_{j}\left(s, y_{s}^{2}, z_{s}^{2}\right)\right|^{2} d s-\frac{1}{2} \int_{t}^{T} \phi_{\varepsilon}^{\prime \prime}\left(\bar{y}_{j}(s)\right)\left|\bar{z}_{j}(s)\right|^{2} d s .
\end{aligned}
$$


Let $\varepsilon \rightarrow 0$, we get

$$
\begin{aligned}
\left|\bar{y}_{j}(t)^{-}\right|^{2}= & \left|\bar{\xi}_{j}^{-}\right|^{2}-\int_{t}^{T} 2 \bar{y}_{j}(s)^{-}\left[f_{j}^{1}\left(s, y_{s}^{1}, z_{s}^{1}\right)-f_{j}^{2}\left(s, y_{s}^{2}, z_{s}^{2}\right)\right] d s \\
& -\int_{t}^{T} 2 \bar{y}_{j}(s)^{-}\left[g_{j}\left(s, y_{s}^{1}, z_{s}^{1}\right)-g_{j}\left(s, y_{s}^{2}, z_{s}^{2}\right)\right] d B_{s}+\int_{t}^{T} 2 \bar{y}_{j}(s)^{-} \bar{z}_{j}(s) d W_{s} \\
& +\int_{t}^{T} \mathbf{I}_{\left(\bar{y}_{j}(s) \leq 0\right)}\left|g_{j}\left(s, y_{s}^{1}, z_{s}^{1}\right)-g_{j}\left(s, y_{s}^{2}, z_{s}^{2}\right)\right|^{2} d s-\int_{t}^{T} \mathbf{I}_{\left(\bar{y}_{j}(s) \leq 0\right)}\left|\bar{z}_{j}(s)\right|^{2} d s .
\end{aligned}
$$

From (i), we have $\xi^{1}-\xi^{2} \geq 0$, a.s., so

$$
E\left|\left(\xi^{1}-\xi^{2}\right)^{-}\right|^{2}=0
$$

Since $\left(y^{1}, z^{1}\right)$ and $\left(y^{2}, z^{2}\right)$ are in $S^{2}\left([0, T] ; R^{k}\right) \times M^{2}\left(0, T ; R^{k \times d}\right)$, it easily follows that

$$
\begin{gathered}
E \int_{t}^{T} \bar{y}_{j}(s)^{-}\left[g_{j}\left(s, y_{s}^{1}, z_{s}^{1}\right)-g_{j}\left(s, y_{s}^{2}, z_{s}^{2}\right)\right] d B_{s}=0 \\
E \int_{t}^{T} \bar{y}_{j}(s)^{-} \bar{z}_{j}(s) d W_{s}=0 .
\end{gathered}
$$

Let

$$
\begin{aligned}
\triangle^{1}=-2 \int_{t}^{T} \bar{y}_{j}(s)^{-} & {\left.\left[f_{j}^{1}\left(s, y_{s}^{1}, z_{s}^{1}\right)-f_{j}^{2}\left(s, y_{s}^{2}, z_{s}^{2}\right)\right] d s\right] } \\
=-2 \int_{t}^{T} \bar{y}_{j}(s)^{-} & {\left[f_{j}^{1}\left(y_{1}^{1}, \ldots, y_{j}^{1}, \ldots, y_{k}^{1}, z_{1}^{1}, \ldots, z_{j}^{1}, \ldots, z_{k}^{1}\right)\right.} \\
& \left.\quad-f_{j}^{1}\left(y_{1}^{1}+\bar{y}_{1}^{-}, \ldots, y_{j}^{2}, \ldots, y_{k}^{1}+\bar{y}_{k^{-}}, z_{1}^{1}, \ldots, z_{j}^{2}, \ldots, z_{k}^{1}\right)\right] d s \\
-2 \int_{t}^{T} \bar{y}_{j}(s)^{-} & {\left[f_{j}^{1}\left(y_{1}^{1}+\bar{y}_{1}^{-}, \ldots, y_{j}^{2}, \ldots, y_{k}^{1}+\bar{y}_{k^{-}}, z_{1}^{1}, \ldots, z_{j}^{2}, \ldots, z_{k}^{1}\right)\right.} \\
& \left.\quad-f_{j}^{2}\left(y_{1}^{2}, \ldots, y_{j}^{2}, \ldots, y_{k}^{2}, z_{1}^{2}, \ldots, z_{j}^{2}, \ldots, z_{k}^{2}\right)\right] d s \\
= & \triangle_{1}^{1}+\triangle_{2^{\prime}}^{1}
\end{aligned}
$$

where $\Delta_{1}^{1}$ is the first integral, and $\Delta_{2}^{1}$ is the second integral. Since

$$
y_{l}^{1}+\bar{y}_{l}^{-} \geq y_{l}^{2}, \quad l \neq j
$$


Journal of Applied Mathematics

and (ii) we have

$$
f_{j}^{1}\left(y_{1}^{1}+\bar{y}_{1}^{-}, \ldots, y_{j}^{2}, \ldots, y_{k}^{1}+\bar{y}_{k}^{-}, z_{1}^{1}, \ldots, z_{j}^{2}, \ldots, z_{k}^{1}\right) \geq f_{j}^{2}\left(y_{1}^{2}, \ldots, y_{j}^{2}, \ldots, y_{k}^{2}, z_{1}^{2}, \ldots, z_{j}^{2}, \ldots, z_{k}^{2}\right)
$$

So $\triangle_{2}^{1} \leq 0$.

From (iii) and Young's inequality, it follows that

$$
\begin{aligned}
\Delta_{1}^{1} \leq & \int_{t}^{T} 2 \bar{y}_{j}^{-} L\left(\left|\bar{y}_{1}^{-}\right|+\cdots+\left|\bar{y}_{j-1}^{-}\right|+\left|\bar{y}_{j}\right|+\left|\bar{y}_{j+1}^{-}\right|+\cdots+\left|\bar{y}_{k}\right|+\left|\bar{z}_{j}\right|\right) d s \\
& =\int_{t}^{T} L\left(2 \bar{y}_{j}^{-}\left|\bar{y}_{1}^{-}\right|+\cdots+2 \bar{y}_{j}^{-}\left|\bar{y}_{j-1}^{-}\right|+2 \bar{y}_{j}\left|\bar{y}_{j}\right|+2 \bar{y}_{j}^{-}\left|\bar{y}_{j+1}\right|+\cdots+2 \bar{y}_{j}^{-}\left|\bar{y}_{k}^{-}\right|+2 \bar{y}_{j}^{-}\left|\bar{z}_{j}\right|\right) d s \\
& =\int_{t}^{T} L\left(2 \bar{y}_{j}^{-}\left|\bar{y}_{1}^{-}\right|+\cdots+2 \bar{y}_{j}\left|\bar{y}_{j-1}^{-}\right|+2\left|\bar{y}_{j}^{-}\right|^{2}+2 \bar{y}_{j}\left|\bar{y}_{j+1}^{-}\right|+\cdots+2 \bar{y}_{j}^{-}\left|\bar{y}_{k}^{-}\right|+2 \bar{y}_{j}\left|\bar{z}_{j}\right|\right) d s \\
& \leq \int_{t}^{T} L\left(\left|\bar{y}_{j}^{-}\right|^{2}+\left|\bar{y}_{1}^{-}\right|^{2}+\cdots+\left|\bar{y}_{j}\right|^{2}+\left|\bar{y}_{j-1}^{-}\right|^{2}+2\left|\bar{y}_{j}\right|^{2}+\left|\bar{y}_{j}^{-}\right|^{2}+\left|\bar{y}_{j+1}\right|^{2}\right. \\
& \left.\quad+\cdots+\left|\bar{y}_{j}^{-}\right|^{2}+\left|\bar{y}_{k}\right|^{2}+\frac{L}{1-\alpha}\left|\bar{y}_{j}^{-}\right|^{2}+\frac{1-\alpha}{L} \mathbf{I}_{\left(\bar{y}_{j} \leq 0\right)}\left|\bar{z}_{j}\right|^{2}\right) d s \\
= & \left.\left(L k+\frac{L^{2}}{1-\alpha}\right) \int_{t}^{T}\left|\bar{y}_{j}^{-}\right|^{2} d s+L \sum_{l=1}^{k} \int_{t}^{T}\left|\bar{y}_{l}^{-}\right|^{2} d s+(1-\alpha) \int_{t}^{T} \mathbf{I}_{\left(\bar{y}_{j} \leq 0\right)}\left|\bar{z}_{j}\right|^{2}\right) d s,
\end{aligned}
$$

where $L>0$ only depends on the Lipschitz constant $C$ in (iii). From (iv) we deduce

$$
\begin{aligned}
\triangle^{2} & =\int_{t}^{T} \mathbf{I}_{\left(\bar{y}_{j}(s) \leq 0\right)}\left|g_{j}\left(s, y_{s}^{1}, z_{s}^{1}\right)-g_{j}\left(s, y_{s}^{2}, z_{s}^{2}\right)\right|^{2} d s \\
& =\int_{t}^{T} \mathbf{I}_{\left.\bar{y}_{j}(s) \leq 0\right)}\left|h_{j}\left(s, y_{j}^{1}(s), z_{j}^{1}(s)\right)-h_{j}\left(s, y_{j}^{2}(s), z_{j}^{2}(s)\right)\right|^{2} d s \\
& \leq \int_{t}^{T} \mathbf{I}_{\left(\bar{y}_{j}(s) \leq 0\right)}\left[C\left|y_{j}^{1}(s)-y_{j}^{2}(s)\right|^{2}+\alpha\left|z_{j}^{1}(s)-z_{j}^{2}(s)\right|^{2}\right] d s \\
& =C \int_{t}^{T}\left|\bar{y}_{j}^{-}\right|^{2} d s+\alpha \int_{t}^{T} \mathbf{I}_{\left(\bar{y}_{j}(s) \leq 0\right)}\left|\bar{z}_{j}\right|^{2} d s .
\end{aligned}
$$

So there $\exists M>0$ only depends on $C, \alpha, k, d$, such that

$$
\Delta^{1}+\triangle^{2} \leq M \sum_{l=1}^{k} \int_{t}^{T}\left|\bar{y}_{l}^{-}\right|^{2} d s+\int_{t}^{T} \mathbf{I}_{\left.\bar{y}_{j}(s) \leq 0\right)}\left|\bar{z}_{j}\right|^{2} d s .
$$


Taking expectation on both sides of (3.10) and noting (3.11)-(3.19), we get

$$
E\left|\bar{y}_{j}(t)^{-}\right|^{2} \leq M E \sum_{l=1}^{k} \int_{t}^{T}\left|\bar{y}_{l}^{-}\right|^{2} d s
$$

We sum from 1 to $k$ and get $\sum_{j=1}^{k} E\left|\bar{y}_{j}(t)^{-}\right|^{2} \leq M k E \sum_{l=1}^{k} \int_{t}^{T}\left|\bar{y}_{l}^{-}\right|^{2} d s$. That is,

$$
E \sum_{j=1}^{k}\left|\bar{y}_{j}(t)^{-}\right|^{2} \leq M k \int_{t}^{T} E \sum_{j=1}^{k}\left|\bar{y}_{j}^{-}\right|^{2} d s
$$

By Gronwall's inequality, it follows that

$$
E \sum_{j=1}^{k}\left|\bar{y}_{j}(t)^{-}\right|^{2}=0, \quad \forall t \in[0, T] . d t-\text { a.s. }
$$

So $\bar{y}_{j}(t) \geq 0 d t \otimes d P$ - a.s. $j=1,2, \ldots, k$.

Example 3.3. If conditions (ii) and (iv) of the theorem are replaced by the following: (ii') for all $j=1,2, \ldots, k, \forall(\omega, t) \in \Omega \times[0, T], y \in R^{k}, z^{1}, z^{2} \in R^{k \times d}, z_{j}^{1}=z_{j}^{2}$

$$
f_{j}^{1}\left(\omega, t, y, z^{1}\right) \geq f_{j}^{2}\left(\omega, t, y, z^{2}\right)
$$

and (iv') for all $(\omega, t, y, z) \in \Omega \times[0, T] \times R^{k} \times R^{k \times d}$,

$$
\left\|g\left(t, y_{1}, z_{1}\right)-g\left(t, y_{2}, z_{2}\right)\right\|^{2} \leq C\left|y_{1}-y_{2}\right|^{2}+\alpha\left\|z_{1}-z_{2}\right\|^{2}
$$

where $C>0,0<\alpha<1$ are two constants. By the following example we can see, the comparison theorem is untenable at the above conditions.

If $m=2, f^{1}(y, z)=f^{2}(y, z)=f(y, z)=A y, g(y, z)=0$, where $A_{2 \times 2}$ is a constant coefficient matrix, obviously $f, g$ satisfy (ii') and (iv'). We consider the following $k$ dimensional BDSDEs: $(0 \leq t \leq T)$

$$
y_{t}^{1}=\xi^{1}+\int_{t}^{T} A y_{s}^{1} d s-\int_{t}^{T} z_{s}^{1} d W_{s}, \quad y_{t}^{2}=\xi^{2}+\int_{t}^{T} A y_{s}^{2} d s-\int_{t}^{T} z_{s}^{2} d W_{s}
$$

then

$$
y_{t}^{1}=E\left[\frac{e^{A(T-t)} \xi^{1}}{\mathcal{F}_{t}^{w}}\right], \quad y_{t}^{2}=E\left[\frac{e^{A(T-t)} \xi^{2}}{\mathcal{F}_{t}^{w}}\right]
$$

Already know $\xi^{1} \geq \xi^{2}, P$ a.s., to be sure $y_{t}^{1} \geq y_{t}^{2}, d t \otimes d P$ a.s., this requires

$$
e^{A(T-t)} \xi^{1} \geq e^{A(T-t)} \xi^{2}, \quad d t \otimes d P \text { a.s., }
$$


then

$$
e^{A(T-t)}\left(\xi^{1}-\xi^{2}\right) \geq 0, \quad d t \otimes d P \text { a.s. }
$$

Let

$$
A=\left[\begin{array}{cc}
0 & -1 \\
1 & 0
\end{array}\right]
$$

then

$$
e^{A(T-t)}=\left[\begin{array}{cc}
\cos (T-t) & \sin (T-t) \\
-\sin (T-t) & \cos (T-t)
\end{array}\right]
$$

Here $f, g$ satisfy (ii') and (iv') does not satisfy (ii) and (iv). Let $T=2 \pi, t \in[0, \pi / 2]$

$$
e^{A(T-t)}=\left[\begin{array}{cc}
\cos t & -\sin t \\
\sin t & \cos t
\end{array}\right]
$$

Obviously we cannot guarantee, for all $\xi^{1} \geq \xi^{2}$, there is $y_{t}^{1} \geq y_{t}^{2}, d t \otimes d P$ a.s.

\section{The Multidimensional BDSDEs with Continuous Coefficients}

In this section, we do not assume a Lipschitz property. Our main interest in this section is to study the multidimensional BDSDEs with continuous coefficients as an application of the comparison theorem obtained in Section 3. First, we give the definition of quasimonotonously increasing mapping [22].

Definition 4.1. A mapping $f: R^{k} \rightarrow R^{k}$ is said to be quasi-monotonously increasing mapping, if for each $j=1,2, \ldots, k, f_{j}(x) \leq f_{j}(y)$ provided $x_{j}=y_{j}$, and $x_{l} \leq y_{l}, l \neq j$.

We give our main result.

Theorem 4.2. Assume $f: \Omega \times[0, T] \times R^{k} \times R^{k \times d} \rightarrow R^{k}$ and $g: \Omega \times[0, T] \times R^{k} \times R^{k \times d} \rightarrow R^{k \times l}$ are jointly measurable functions and satisfy.

(1) There exists $r(\omega, t, y, \gamma): \Omega \times[0, T] \times R^{k} \times R^{d} \rightarrow R^{k}$, such that for all $(\omega, t, y, z) \in$ $\Omega \times[0, T] \times R^{k} \times R^{k \times d}$,

$$
f_{j}(\omega, t, y, z)=r_{j}\left(\omega, t, y, z_{j}\right)
$$

(2) Linear growth: for all $(\omega, t, y, z) \in \Omega \times[0, T] \times R^{k} \times R^{k \times d}, \exists K>0$, such that

$$
|f(\omega, t, y, z)| \leq K(1+|y|+\|z\|) \text {. }
$$

(3) Continuous: for fixed $\omega$ and $t, f(\omega, t, \cdot, \cdot)$ is continuity. 
(4) Quasimonotonously increasing: for fixed $\omega, t$ and $z, f(\omega, t, \cdot, z)$ is quasi-monotonously increase, that is for all $j=1,2, \ldots, k$, such that

$$
f_{j}\left(\omega, t, y^{1}, z\right) \geq f_{j}\left(\omega, t, y^{2}, z\right), \quad \forall y^{1}, y^{2} \in R^{k}, \quad y_{j}^{1}=y_{j}^{2}, \quad y_{l}^{1} \geq y_{l}^{2}, \quad l \neq j ;
$$

(5) Function $g$ satisfies the (iv) of Theorem 3.2, that is for all $(\omega, t, y, z) \in \Omega \times[0, T] \times R^{k} \times$ $R^{k \times d}, \exists h(\omega, t, \mu, v): \Omega \times[0, T] \times R \times R^{d} \rightarrow R$ such that $g_{j}(\omega, t, y, z)=h_{j}\left(\omega, t, y_{j}, z_{j}\right)$ and

$$
\left|h_{j}\left(t, y_{j}^{1}, z_{j}^{1}\right)-h_{j}\left(t, y_{j}^{2}, z_{j}^{2}\right)\right|^{2} \leq C\left|y_{j}^{1}-y_{j}^{2}\right|^{2}+\alpha\left|z_{j}^{1}-z_{j}^{2}\right|^{2}
$$

where $C>0,0<\alpha<1$ are two constants. Then, if $\xi \in L^{2}\left(\Omega, \Psi_{T}, P ; R^{k}\right), B D S D E(2.5)$ has a solution $(y, z) \in S^{2}\left([0, T] ; R^{k}\right) \times M^{2}\left(0, T ; R^{k \times d}\right)$.

We give a lemma, this method was first introduced by [23] Barlow and Perkins. For fixed $(\omega, t) \in \Omega \times[0, T]$, we define the sequence $f^{n}(\omega, t, y, z): \Omega \times[0, T] \times R^{k} \times R^{k \times d} \rightarrow R^{k}$ as the following: $j=1,2, \ldots, k$.

$$
f_{j}^{n}(\omega, t, y, z) \doteq \inf _{x \in R^{k}, \gamma \in R^{k \times d}}\left\{f_{j}(\omega, t, x, \gamma)+\sum_{l=1, l \neq j}^{k} n\left(y_{l}-x_{l}\right)^{+}+n\left|y_{j}-x_{j}\right|+n\|z-\gamma\|\right\} .
$$

\section{Lemma 4.3.}

Let $n \geq K$ and $f^{n}$ be define as in (4.5), then $f^{n}$ satisfies the following properties.

(1) For all $(\omega, t, y, z) \in \Omega \times[0, T] \times R^{k} \times R^{k \times d}$,

$$
f_{j}^{n}(\omega, t, y, z) \leq f_{j}(\omega, t, y, z)
$$

(2) Uniformly linear growth: for all $(\omega, t, y, z) \in \Omega \times[0, T] \times R^{k} \times R^{k \times d}$,

$$
\left|f_{j}^{n}(\omega, t, y, z)\right| \leq K\left(1+|y|+\left|z_{j}\right|\right)
$$

(3) Quasimonotonous increase with respect to y: for all $(\omega, t) \in \Omega \times[0, T], z^{1}, z^{2} \in R^{k \times d}$

$$
f_{j}^{n}\left(\omega, t, y^{1}, z^{1}\right) \geq f_{j}^{n}\left(\omega, t, y^{2}, z^{2}\right), \quad \forall y_{j}^{1}=y_{j}^{2}, z_{j}^{1}=z_{j}^{2}, y_{l}^{1} \geq y_{l}^{2}, l \neq j
$$

(4) Monotonous increase with respect to $n$ : for all $(\omega, t, y, z) \in \Omega \times[0, T] \times R^{k} \times R^{k \times d}$,

$$
f_{j}^{n}(\omega, t, y, z) \leq f_{j}^{n+1}(\omega, t, y, z)
$$

(5) Lipschitz condition: for all $(\omega, t) \in \Omega \times[0, T],\left(y^{1}, z^{1}\right),\left(y^{2}, z^{2}\right) \in R^{k} \times R^{k \times d}$,

$$
\left|f_{j}^{n}\left(\omega, t, y^{1}, z^{1}\right)-f_{j}^{n}\left(\omega, t, y^{2}, z^{2}\right)\right| \leq n\left(\left|y^{1}-y^{2}\right|+\left\|z^{1}-z^{2}\right\|\right),
$$


Journal of Applied Mathematics

(6) Strong convergence: for all $(\omega, t) \in \Omega \times[0, T],(y, z),\left(y^{n}, z^{n}\right) \in R^{k} \times R^{k \times d}, n=1,2, \ldots$, if $y^{n} \rightarrow y, z^{n} \rightarrow z$, when $n \rightarrow \infty$. Then

$$
f^{n}\left(\omega, t, y^{n}, z^{n}\right) \longrightarrow f(\omega, t, y, z)
$$

We consider the following $k$-dimensional BDSDEs

$$
\begin{gathered}
y_{t}^{n}=\xi+\int_{t}^{T} f^{n}\left(s, y_{s}^{n}, z_{s}^{n}\right) d s+\int_{t}^{T} g\left(s, y_{s}^{n}, z_{s}^{n}\right) d B_{s}-\int_{t}^{T} z_{s}^{n} d W_{s}, \\
U_{t}=\xi+\int_{t}^{T} F\left(s, U_{s}, V_{s}\right) d s+\int_{t}^{T} g\left(s, U_{s}, V_{s}\right) d B_{s}-\int_{t}^{T} V_{s} d W_{s},
\end{gathered}
$$

where $F: \Omega \times[0, T] \times R^{k} \times R^{k \times d} \rightarrow R^{k}$, and

$$
F_{j}(\omega, t, y, z)=K\left(1+|y|+\left|z_{j}\right|\right), \quad j=1,2, \ldots, k
$$

From Lemma 4.3, for all $(\omega, t) \in \Omega \times[0, T],\left(y^{1}, z^{1}\right),\left(y^{2}, z^{2}\right) \in R^{k} \times R^{k \times d}, y_{j}^{1}=y_{j}^{2}, z_{j}^{1}=z_{j}^{2}$ $y_{l}^{1} \geq y_{l}^{2}, l \neq j$, we have

$$
\begin{aligned}
f_{j}^{n}\left(t, y^{2}, z^{2}\right) & \leq f_{j}^{n+1}\left(t, y^{2}, z^{2}\right) \\
& \leq f_{j}^{n+1}\left(t, y^{1}, z^{1}\right) \\
& \leq K\left(1+\left|y^{1}\right|+\left|z_{j}^{1}\right|\right) \\
& =F_{j}\left(t, y^{1}, z^{1}\right), \quad n=K, K+1, \ldots
\end{aligned}
$$

From Pardoux and Peng [8], BDSDE (4.12) and (4.13) has a unique solution

$$
\left(y^{n}, z^{n}\right) \in S^{2}\left([0, T] ; R^{k}\right) \times M^{2}\left(0, T ; R^{k \times d}\right), \quad(U, V) \in S^{2}\left([0, T] ; R^{k}\right) \times M^{2}\left(0, T ; R^{k \times d}\right)
$$

From the comparison Theorem 3.2, for all $n \geq m \geq K$,

$$
y_{j}^{m}(t) \leq y_{j}^{n}(t) \leq U_{j}(t), \quad d t \otimes d P-\text { a.s. }
$$

Lemma 4.4. There exists constant $A>0$ depending only on $K, C, \alpha, T$ and $\xi$, such that

$$
\forall n \geq K, \quad\left\|y^{n}\right\|_{S^{2}} \leq A, \quad\left\|z^{n}\right\|_{M^{2}} \leq A, \quad\|U\|_{S^{2}} \leq A, \quad\|V\|_{M^{2}} \leq A .
$$


Lemma 4.5. $\left\{\left(y^{n}, z^{n}\right)\right\}_{n=1}^{\infty}$ converges in $S^{2}\left([0, T] ; R^{k}\right) \times M^{2}\left(0, T ; R^{k \times d}\right)$.

The proof of the Lemmas 4.4 and 4.5 is similar to Lemma 4.2 and Lemma 4.3 in [19], so we omit it.

We give the proof of Theorem 4.2.

Proof. For all $n \geq n_{0} \geq K$, we have $y^{n_{0}} \leq y^{n} \leq U$, and $y^{n}$ converges in $S^{2}\left([0, T] ; R^{k}\right), d t \otimes d P-$ a.s. to $y \in S^{2}\left([0, T] ; R^{k}\right)$.

On the other hand, since $z^{n}$ converges in $M^{2}\left(0, T ; R^{k \times d}\right)$ to $z$, we can assume, choosing a subsequence if needed, that $z^{n} \rightarrow z d t \otimes d P-$ a.s. and $\bar{G}_{t}=\sup _{n}\left\|z_{t}^{n}\right\|$ is $d t \otimes d P$ integrable. Therefore, from (3.11) and (2.5) of the Lemma 4.3, we get for almost all $\omega$,

$$
\begin{aligned}
f^{n}\left(t, y_{t}^{n}, z_{t}^{n}\right) & \longrightarrow f\left(t, y_{t}, z_{t}\right), \quad(n \rightarrow \infty) d t-\text { a.e. } \\
\left|f^{n}\left(t, y_{t}^{n}, z_{t}^{n}\right)\right| & \leq K\left(1+\sup _{n}\left|y_{t}^{n}\right|+\sup _{n}\left\|z_{t}^{n}\right\|\right) \\
& =K\left(1+\sup _{n}\left|y_{t}^{n}\right|+\bar{G}_{t}\right) \in L^{1}([0, T] ; d t) .
\end{aligned}
$$

Thus, for almost $\omega$ and uniformly in $t$, it holds that

$$
\int_{t}^{T} f^{n}\left(s, y_{s}^{n}, z_{s}^{n}\right) d s \longrightarrow \int_{t}^{T} f\left(s, y_{s}, z_{s}\right) d s, \quad(n \rightarrow \infty)
$$

From the continuity properties of the stochastic integral, it follows that

$$
\begin{aligned}
& \sup _{0 \leq t \leq T}\left|\int_{t}^{T} z_{s}^{n} d W_{s}-\int_{t}^{T} z_{s} d W_{s}\right| \longrightarrow 0 \text { in probability, } \\
& \sup _{0 \leq t \leq T}\left|\int_{t}^{T} g\left(s, y_{s}^{n}, z_{s}^{n}\right) d B_{s}-g\left(s, y_{s}, z_{s}\right) d B_{s}\right| \longrightarrow 0 \text { in probability. }
\end{aligned}
$$

Choosing, again, a subsequence, we can assume that the above convergence is P-a.s. Finally,

$$
\begin{aligned}
\left|y_{t}^{n}-y_{t}^{m}\right| \leq \int_{t}^{T} & \left|f^{n}\left(s, y_{s}^{n}, z_{s}^{n}\right)-f^{m}\left(s, y_{s}^{m}, z_{s}^{m}\right)\right| d s \\
& +\left|\int_{t}^{T}\left(g\left(s, y_{s}^{n}, z_{s}^{n}\right)-g\left(s, y_{s}^{m}, z_{s}^{m}\right)\right) d B_{s}\right|+\left|\int_{t}^{T}\left(z_{s}^{n}-z_{s}^{m}\right) d W_{s}\right|
\end{aligned}
$$


and taking limits in $m$ and supremum over $t$, we get

$$
\begin{aligned}
\sup _{0 \leq t \leq T}\left|y_{t}^{n}-y_{t}\right| \leq & \int_{0}^{T}\left|f^{n}\left(s, y_{s}^{n}, z_{s}^{n}\right)-f\left(s, y_{s}, z_{s}\right)\right| d s \\
& +\sup _{0 \leq t \leq T}\left|\int_{t}^{T}\left(g\left(s, y_{s}^{n}, z_{s}^{n}\right)-g\left(s, y_{s}, z_{s}\right)\right) d B_{s}\right| \\
& +\sup _{0 \leq t \leq T}\left|\int_{t}^{T}\left(z_{s}^{n}-z_{s}\right) d W_{s}\right| P-\text { a.s. }
\end{aligned}
$$

From which it follows that $y^{n}$ converges uniformly in $t$ to $y$ (in particular, $y$ is a continuous process). Note that $y^{n}$ is monotone, therefore, we actually have the uniform convergence for the entire sequence and not just for a subsequence. Taking limits in (4.12), we deduce that $(y, z)$ is the solution of $(2.5)$.

From the comparison theorem, we can get a result.

Lemma 4.6. $y$ is the minimal solution of BDSDE (2.5).

Proof. If let $(\widehat{y}, \widehat{z}) \in S^{2}\left([0, T] ; R^{k}\right) \times M^{2}\left(0, T ; R^{k \times d}\right)$ be any solution of $(2.5)$, from the comparison Theorem 3.2, we get that $y^{n} \leq \widehat{y}$, for all $n \in N$, and therefore $y \leq \hat{y}$. That is, $y$ is the minimal solution of BDSDE (2.5).

\section{Acknowledgment}

This work is supported the Nature Science Foundation of Shandong Province of China (Grant no. ZR2010AL014).

\section{References}

[1] É. Pardoux and S. G. Peng, "Adapted solution of a backward stochastic differential equation," Systems $\mathcal{E}$ Control Letters, vol. 14, no. 1, pp. 55-61, 1990.

[2] S. G. Peng, "Probabilistic interpretation for systems of quasilinear parabolic partial differential equations," Stochastics and Stochastics Reports, vol. 37, no. 1-2, pp. 61-74, 1991.

[3] É. Pardoux and S. Peng, "Backward stochastic differential equations and quasilinear parabolic partial differential equations," in Stochastic Partial Differential Equations and Their Applications, B. L. Rozovskii and R. Sowers, Eds., vol. 176 of Lecture Notes in Control and Inform. Sci., pp. 200-217, Springer, Berlin, Germany, 1992.

[4] N. El Karoui, S. Peng, and M. C. Quenez, "Backward stochastic differential equations in finance," Mathematical Finance, vol. 7, no. 1, pp. 1-71, 1997.

[5] S. Hamadene and J.-P. Lepeltier, "Zero-sum stochastic differential games and backward equations," Systems \& Control Letters, vol. 24, no. 4, pp. 259-263, 1995.

[6] J. Ma and J. Yong, Forward-Backward Stochastic Differential Equations and Their Applications, vol. 1702 of Lecture Notes in Mathematics, Springer, Berlin, Germany, 1999.

[7] C. Geiß and R. Manthey, "Comparison theorems for stochastic differential equations in finite and infinite dimensions," Stochastic Processes and Their Applications, vol. 53, no. 1, pp. 23-35, 1994.

[8] E. Pardoux and S. G. Peng, "Backward doubly stochastic differential equations and systems of quasilinear SPDEs," Probability Theory and Related Fields, vol. 98, no. 2, pp. 209-227, 1994.

[9] Q. Zhang and H. Zhao, "Stationary solutions of SPDEs and infinite horizon BDSDEs," Journal of Functional Analysis, vol. 252, no. 1, pp. 171-219, 2007. 
[10] B. Zhu and B. Han, "Backward doubly stochastic differential equations with infinite time horizon," Applicationes Mathematicae. Accepted.

[11] V. Bally and A. Matoussi, "Weak solutions for SPDEs and backward doubly stochastic differential equations," Journal of Theoretical Probability, vol. 14, no. 1, pp. 125-164, 2001.

[12] R. Buckdahn and J. Ma, "Stochastic viscosity solutions for nonlinear stochastic partial differential equations. I," Stochastic Processes and Their Applications, vol. 93, no. 2, pp. 181-204, 2001.

[13] R. Buckdahn and J. Ma, "Stochastic viscosity solutions for nonlinear stochastic partial differential equations. II," Stochastic Processes and Their Applications, vol. 93, no. 2, pp. 205-228, 2001.

[14] E. Pardoux, "Stochastic partial differential equations," Fudan lecture notes, 2007.

[15] S. Peng and Y. Shi, "A type of time-symmetric forward-backward stochastic differential equations," Comptes Rendus Mathématique. Académie des Sciences. Paris, vol. 336, no. 9, pp. 773-778, 2003.

[16] B. Zhu and B. Y. Han, "Backward doubly stochastic differential equations with non-Lipschitz coefficients," Acta Mathematica Scientia A, vol. 28, no. 5, pp. 977-984, 2008.

[17] B. Boufoussi, J. Van Casteren, and N. Mrhardy, "Generalized backward doubly stochastic differential equations and SPDEs with nonlinear Neumann boundary conditions," Bernoulli, vol. 13, no. 2, pp. 423-446, 2007.

[18] L. Hu and Y. Ren, "Stochastic PDIEs with nonlinear Neumann boundary conditions and generalized backward doubly stochastic differential equations driven by Lévy processes," Journal of Computational and Applied Mathematics, vol. 229, no. 1, pp. 230-239, 2009.

[19] Y. Shi, Y. Gu, and K. Liu, "Comparison theorems of backward doubly stochastic differential equations and applications," Stochastic Analysis and Applications, vol. 23, no. 1, pp. 97-110, 2005.

[20] G. Da Prato and J. Zabczyk, Stochastic Equations in Infinite Dimensions, vol. 44 of Encyclopedia of Mathematics and Its Applications, Cambridge University Press, Cambridge, UK, 1992.

[21] D. Nualart and É. Pardoux, "Stochastic calculus with anticipating integrands," Probability Theory and Related Fields, vol. 78, no. 4, pp. 535-581, 1988.

[22] S. Assing and R. Manthey, "The behavior of solutions of stochastic differential inequalities," Probability Theory and Related Fields, vol. 103, no. 4, pp. 493-514, 1995.

[23] M. T. Barlow and E. Perkins, "One-dimensional stochastic differential equations involving a singular increasing process," Stochastics, vol. 12, no. 3-4, pp. 229-249, 1984. 


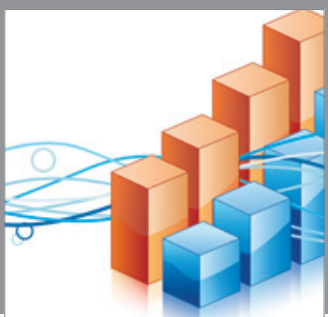

Advances in

Operations Research

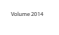

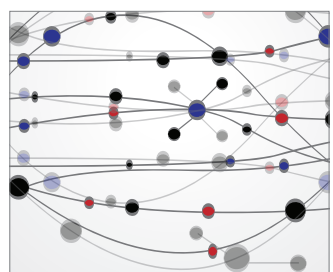

\section{The Scientific} World Journal
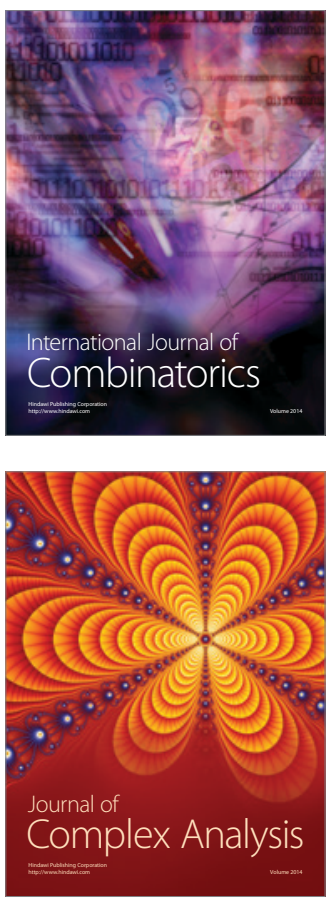

International Journal of

Mathematics and

Mathematical

Sciences
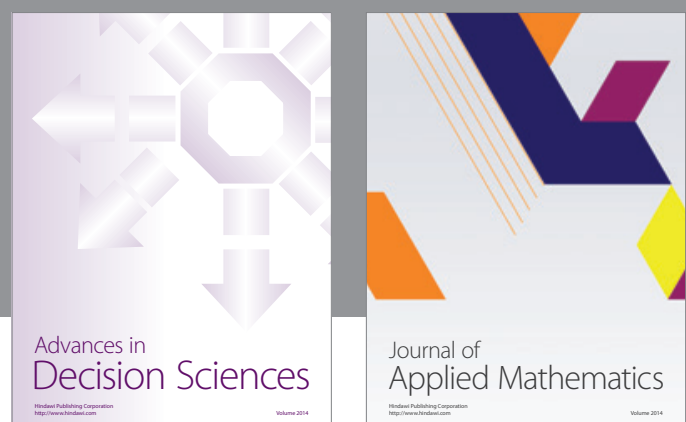

Journal of

Applied Mathematics
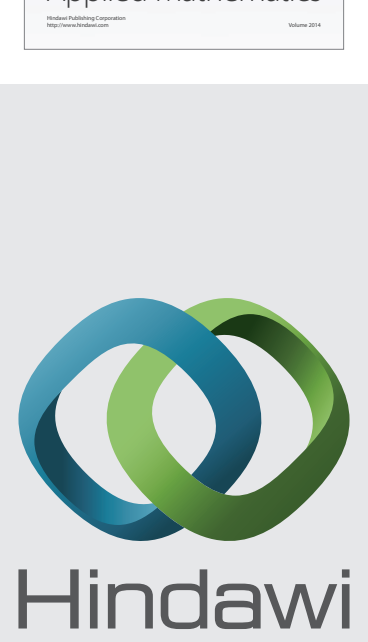

Submit your manuscripts at http://www.hindawi.com
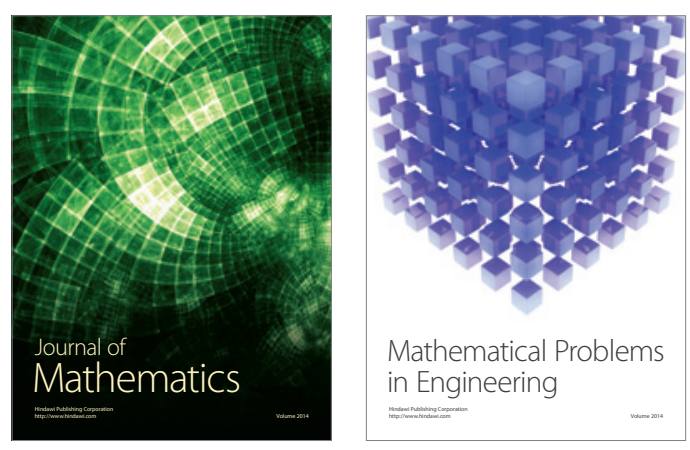

Mathematical Problems in Engineering
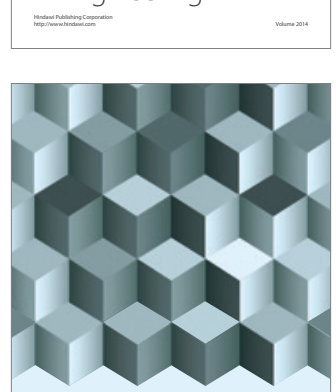

Journal of

Function Spaces
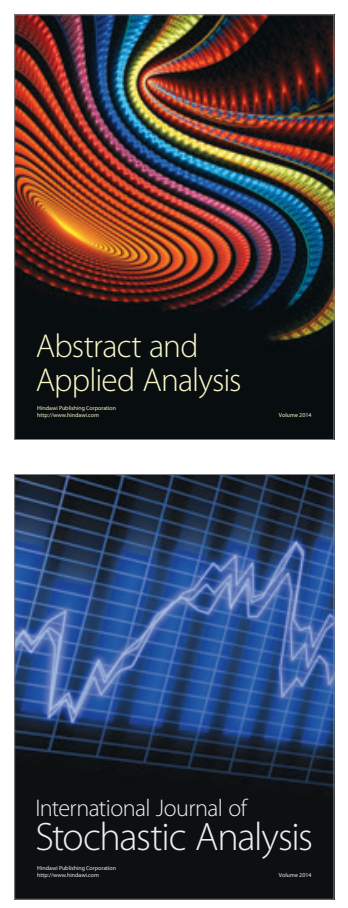

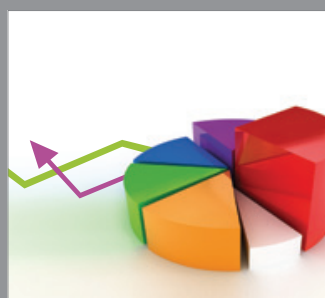

ournal of

Probability and Statistics

Promensencen
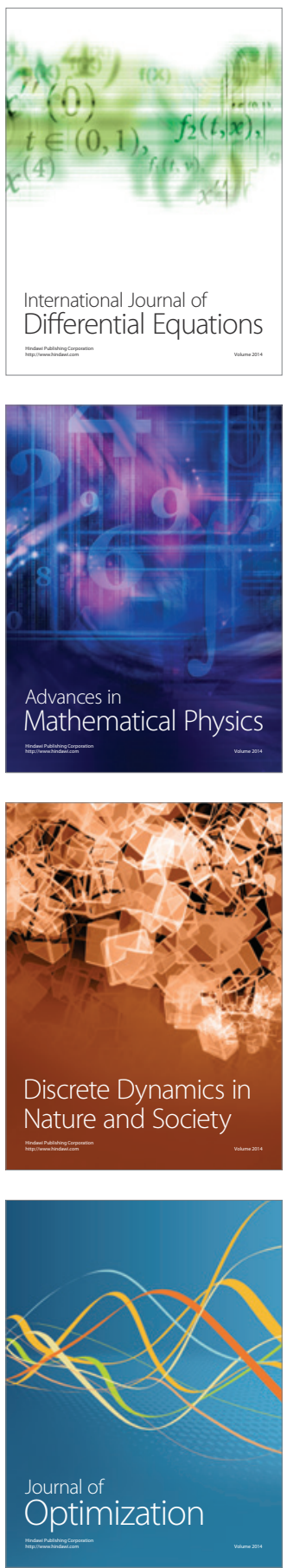